\title{
In search of details of patient teaching in nursing documentation - an analysis of patient records in a medical ward in Sweden
}

\author{
Febe Friberg PhD, RN \\ Senior lecturer, School of Health Sciences, University College of Borås, Borås, Sweden \\ Anne-Louise Bergh MSC, RN \\ Lecturer, School of Health Sciences, University College of Borås, Borås, Sweden \\ Margret Lepp PhD, RN \\ Senior lecturer, School of Health Sciences, University College of Borås, Borås, Sweden
}

Submitted for publication: 24 August 2005

Accepted for publication: 30 August 2005

\author{
Correspondence: \\ Febe Friberg \\ School of Health Sciences \\ University College of Borås \\ Allégatan 1 \\ 50190 Borås \\ Sweden \\ Telephone: $+46-33174747$ \\ E-mail: febe.friberg@hb.se
}

FRIBERG F, BERGH A-L \& LEPP M (2006) Journal of Clinical Nursing 15, 15501558

In search of details of patient teaching in nursing documentation - an analysis of patient records in a medical ward in Sweden

Aim. The aim of this study was to identify terms and expressions indicating patients' need for knowledge and understanding, as well as nurses' teaching interventions, as documented in nursing records.

Background. Previous international studies have shown that nursing documentation is often deficient in terms of recording patient teaching.

Methods. Patient records $(N=35)$ were collected in a general medical ward in a hospital in Sweden. The data contain 206 days of nursing documentation. The records were analysed with regard to content and structure. Terms and expressions indicating patients' need for knowledge and understanding and terms and expressions indicating nurses' teaching activities were analysed.

Results. The results showed that patients' need for knowledge is implicitly indicated by conceptions and experiences as well as questions. Furthermore, nurses' implicit teaching interventions consist of information, motivating conversations, explanations, instructions and setting expectations. However, the content and structure of the pedagogical activities in the patient records are fragmented and vague.

Relevance to clinical practice. Efforts must be directed towards elaborating upon the above-mentioned terms and expressions as indications of patients' need for knowledge and nurses' teaching interventions. Moreover, these terms and expressions must be recognized and acknowledged.

Key words: need for knowledge, nursing, nursing documentation, patient education, patient records, teaching intervention

\section{Introduction}

Patient teaching is considered to be a regular nursing activity (Redman 1993, Falvo 1994, Coates 1999). However, a number of studies reveal that patient teaching is not properly documented in patient records (Ehnfors \& Smedby 1993, Casey 1995, Hale et al. 1997, Turner et al. 1999, Ehrenberg et al. 2001, Marcum et al. 2002, Karkkainen \& Eriksson 2003). In Sweden, nurses are obliged by law to record care (SFS 1985, SOSFS 1993), of which teaching activities are 
regarded as a component. Considerable research efforts have been devoted to documentation at a general level (Ehnfors et al. 1991, Ehrenberg et al. 1996), nurses' views of documenting nursing care in specific settings (Martin et al. 1999, Björvell et al. 2003) and documentation pertaining to specific patient groups (Ekman \& Ehrenberg 2002, Idvall \& Ehrenberg 2002). There are also a number of studies that reveal inconsistencies between documentation and patients' experiences of their condition and nursing care (Adamsen \& Tewes 2000, Cadd et al. 2000, Ekman \& Ehrenberg 2002). These studies demonstrate that patients' preferences are not always mentioned in the documentation. Furthermore, a discrepancy has been found between nurses' experiences of the care provided and the documentation (Hale et al. 1997, Adamsen \& Tewes 2000, Idvall \& Ehrenberg 2002). According to these studies, patients' problems tend to be under-reported in nursing records. Karkkainen and Eriksson (2003) stated that patients' need for knowledge tends to be underestimated in nursing documentation. Given the fact that nursing documentation should realistically reflect the care provided as well as promoting individualized nursing care, patient safety and continuity of care, it is necessary to examine the documentation of patient teaching. In conclusion, there seems to be a lack of knowledge about how to express patient teaching in nursing documentation.

\section{Patient teaching in theory and practice}

To create an understanding of the documentation of patient teaching, the concept of 'patient teaching' must be elucidated to provide a conclusive definition, as several definitions exist (Coates 1999). It should be noted that different terms are used synonymously, such as 'teaching', 'instructions' and 'education' (Falvo 1994). However, the term 'education' is often used in a comprehensive sense. Embedded in these definitions are paradigmatic assumptions that influence teaching activities. For example, Redman's (1993, p. 10) definition of patient education reveals a focus on behavioural and cognitive aspects: 'the deliberative process of creating behavioural and cognitive change in patients'. In another definition (Coutts \& Hardy 1992, p. 47), patient teaching is described more comprehensively as the process of helping or enabling a patient to learn. When exploring nurses' documentation of teaching activities, it is important to note that informal (spontaneous) activities occur in addition to formal (planned) teaching activities (Milazzo 1980, Gregor 2001, Barber-Parker 2002). The major part of patient education research concerns formal teaching. Theories such as the selfefficacy theory, which refers to an individual's belief about his/her ability to perform well (Bandura 1977) and the Health
Belief Model (Janz \& Becker 1984), which concerns patients' perceived susceptibility and the perceived severity of their condition, are often used as theoretical guidelines in education programmes.

Everyday nursing duties mainly consist of teaching activities of an informal nature (Gregor 2001). However, with a few exceptions, informal teaching has been poorly studied. To identify patient teaching, Gregor (2001) investigated the interaction between patients and nurses in a surgical ward in Canada. Six different types of interaction constituting patient teaching emerged: questions, explanations, information, instructions, setting expectations and demonstrating correct modes of conduct. In another study (Friberg 2001), observations of nurse-patient encounters in a medical ward in Sweden were studied to describe informal patient teaching. Based on the field notes, the following teaching strategies, used by nurses, were identified: observing patients' actions and words, asking questions and waiting for the patient to ask questions, providing information, demonstrating modes of conduct, providing explanations, exhorting and giving reasons. The results of Friberg (2001) and Gregor (2001) show similarities concerning strategies used by nurses in teaching situations.

Furthermore, Friberg (2001) identified strategies used by patients for seeking knowledge and gaining understanding, such as observing nurses' words and actions, asking direct (explicit) or indirect (implicit) questions, seeking confirmation of previous experiences and conceptions and interpreting their own bodily expressions to understand the disease and its consequences. Moreover, patients asked other persons as a means of acquiring knowledge and understanding about their condition. Finally, they also searched for information in specialized literature pertaining to their disease. These strategies reveal something about patients' attempts to create knowledge in everyday nursing situations.

As the situation to be documented is a part of everyday nursing practice, it is necessary to clarify the meaning of an informal or spontaneous situation. An informal teaching situation originates in an encounter between nurse and patient, where the patient starts a conversation with the intention of obtaining knowledge about the illness or disease and/or its consequences for his/her present situation or life circumstances. The nurse can also initiate an informal teaching situation by making a comment with the purpose of increasing the patient's knowledge about his/her illness or disease and/or its consequences for the present situation or life circumstances. In the present study, the concept of patient teaching is used in a comprehensive sense and refers to a situation that enables learning and includes both informal and formal teaching activities. 
However, it is difficult to recognize patient teaching in nursing documentation. In this study, the strategies used by nurses in teaching situations (see Friberg 2001, Gregor 2001) can serve as a guide in the search for evidence of teaching interventions in nursing documentation. In the same way, Friberg's (2001) findings concerning patients' strategies for seeking knowledge and understanding can guide the search for such documentary evidence. It is assumed that, to obtain a basis for decisions about appropriate educational interventions, the patients' need for knowledge must be assessed. Thus, a structure that fits into the nursing process is necessary (Yura \& Walsh 1988).

\section{Aim}

The aim of this study was to identify terms and expressions indicating patients' need for knowledge and understanding as well as nurses' teaching interventions as documented in nursing records.

\section{Methods}

\section{Context and data collection}

Patient records were collected from 35 patients (22 women and 13 men, between 35 and 84 years of age) during a fieldwork study on informal patient teaching in a general medical ward (Friberg 2001). In total, the data contain 206 days (one day was counted as 24 hours) of nursing documentation. The reasons for admission to the ward were various problems such as heart failure, liver disease, gastrointestinal diseases, kidney disease, asthma and stroke. The number of days in hospital ranged from 2-23.5 (mean $5 \cdot 9$ days). The data were analysed between September 2003 and April 2004 by the first and second authors.

The collected nursing documentation was organized in accordance with the VIPS model, a model for nursing documentation in patient records that is commonly used in
Sweden and has been used in other European countries (Ehnfors et al. 1991, Ehrenberg et al. 1996). VIPS is an acronym for the Swedish spelling of four key concepts: wellbeing, integrity, prevention and safety. The model consists of keywords on two levels. The first level follows the structure of the nursing process model with the key words: nursing history, nursing status, nursing diagnosis, goal, nursing intervention, nursing outcome and nursing discharge notes. The second level of key words consists of specific terms related to nursing history (examples of key words are Reason for contact/Admission, Health history/Care experience, Current care), nursing status (examples of key words are Communication, Cognition/Development) and nursing intervention (examples of key words are Participation, Information/Education, Support). Accordingly, some second level key words related to nursing status and nursing intervention have pedagogical meaning. When the expression 'nursing status' is used in the study, we refer to the first order key word as 'patient's health situation and condition that influence the present nursing care at the time of contact and continuing throughout the care episode' (Ehrenberg et al. 1996). We assume that, if the nursing process is followed, patients' need for knowledge should be documented under the heading 'nursing status' and nurses' teaching interventions - under the heading 'nursing interventions'.

\section{Data analysis}

The analysis of the documentation was divided into two parts: content and structure analysis.

\section{Analysis of content}

The analysis of content of the documentation was guided by a protocol (Table 1) based on the empirical findings of Friberg (2001) and Gregor (2001). The protocol consists of two essential aspects concerning the documentation of patient teaching; a description of patients' need for knowledge and a description of nurses' teaching interventions.

Table 1 Protocol used to guide the analysis of the content of the documentation

So as to identify patients' need for knowledge, the documentation contains notes about:

- Patients posing implicit (embedded) or explicit (clearly formulated) questions

- Patients' experiences or conceptions of the illness, health situation, etc.

- Patients interpreting bodily expressions so as to understand the disease and its consequences

- Patients asking persons other than the nurse

- Patients reading relevant literature, consulting the Internet, etc.
So as to identify nurses' teaching interventions, the documentation contains notes about:

- Nurses posing questions

- Nurses providing information

- Nurses providing explanations

- Nurses setting expectations

- Nurses exhibiting a mode of conduct

- Nurses giving reasons 
The strategies used in everyday nursing practice can, therefore, guide the search for details in nursing documentation. Thus, the protocol was used as a screen or guide in the search for references to patient teaching in the documentation.

During the analysis, all patient records were read to gain an in-depth understanding of the content. After the first reading, the recorded content was studied for the purpose of identifying terms and expressions based on the following questions: what terms and expressions of pedagogical relevance concerning patients' need for knowledge and understanding appear in the records? What terms and expressions of pedagogical relevance concerning teaching interventions are employed in the records? The term 'pedagogy' is used as a comprehensive expression for teaching and learning activities in nursing situations (Van Manen 1991). 'Pedagogical relevance' is understood as content related to the areas described in the protocol (see Table 1). Both clear expressions (manifest content) and those that required interpretation (latent content) were identified and listed (Graneheim \& Lundman 2004). Thereafter, different areas connected to patients' needs for knowledge and nurses' teaching interventions were formulated.

\section{Analysis of structure}

A spreadsheet was designed to obtain an overview of the structure of the documentation. Patient record numbers were noted on the horizontal axis and keywords from the VIPS model (nursing history, status, diagnosis, goals, interventions, outcome and discharge notes) on the vertical axis. The content of the records of pedagogical relevance was organized in accordance with the structure of the spreadsheet. A protocol developed by Ehnfors and Smedby (1993) based on the steps of the nursing process was used (see also Idvall \& Ehrenberg 2002, p. 736-737) to analyse the clarity of structure of the documentation in terms of patients' need for knowledge and nurses' teaching interventions. The protocol consists of the following five levels:

1 The problem is described or interventions planned or have been implemented.

2 The problem is described and interventions are planned or have been implemented.

3 The problem is described and interventions are planned or have been implemented. The nursing outcome is noted.

4 The problem is described, interventions are planned and have been implemented. The nursing outcome is noted.

5 All steps that form a part of the nursing process are recorded (including nursing history, diagnosis, goals and discharge notes). There is an adequate description of the problem.
Each identified item of pedagogical relevance was explored following the levels described above.

The study was approved by the Research Ethics Committee at the Faculty of Medicine, Göteborg University, Sweden. Permission to access the nursing records was obtained from the patients concerned and the medical director of the department. All personal details were removed to ensure that the patients could not be identified, thus guaranteeing confidentiality.

\section{Results}

Areas identified indicating patients' need for knowledge, areas identified indicating nurses' teaching interventions and the structure of the documentation are presented in the results.

\section{Areas identified that indicate patients' need for knowledge}

Three areas that indicate patients' need for knowledge were identified in the documentation:

- Patients' questions

- Patients' conceptions

- Experiences of bodily changes

\section{Patients' questions}

Patients' questions are noted on 12 occasions in the documentation and originated from 12 different patients, (Table 2). Notes about patients' questions concern planned treatments, medication, medical examinations and tests of different kinds. Other questions concern the patient's future. The questions can have an explicit character, e.g.:

The patient shows a bruise of $2 \times 2 \mathrm{~cm}$ on her right underarm and asks if it was caused by the medication. (record 21)

Table 2 Number of occurrences of areas of pedagogical relevance in relation to time of day

\begin{tabular}{lcclc}
\hline & Daytime & Evenings & Nighttime & Total \\
\hline Patients' questions & 8 & 2 & 2 & 12 \\
Patients' conceptions & 5 & 5 & 1 & 11 \\
$\begin{array}{l}\text { Patients' experiences of } \\
\text { bodily changes }\end{array}$ & 3 & 3 & 1 & 7 \\
$\quad$ Nurses' information & 11 & 4 & 0 & 15 \\
$\quad$ Nurses motivating & 5 & 0 & 0 & 5 \\
$\quad$ conversation & & & & \\
$\quad$ Nurses' explanations & 0 & 2 & 3 & 5 \\
$\quad$ Nurses' instructions & 1 & 1 & 1 & 3 \\
$\quad$ Nurses setting & 1 & 2 & 0 & 3 \\
$\quad$ expectations & & & & \\
$\quad$ Total & 34 & 19 & 8 & \\
\hline
\end{tabular}


Here, the term 'ask' was used by the nurse. Another term used in the documentation was 'wonder'. Questions can also have a more implicit character such as the following:

Weeps and worries about the future. (record 27)

The expression 'weeps and worries about the future' indicates a conversation about what will happen after discharge from hospital. However, the conversation is not documented. In this record the intervention consisted of the administration of sedative medication and 'a short chat'. The intervention was evaluated as having a 'beneficial effect'. Implicit questions occurred on four occasions during the 206 days. It is worth noting that two implicit questions occurred during the night. On three occasions, administration of sedative medication was recorded as a nursing intervention. On two other occasions, questions from the patients' relatives were noted.

\section{Patients' conceptions}

Entries in the documentation about patients' conceptions were identified on 11 occasions during the 206 days and concerned nine patients (Table 2). In one patient record, conceptions about the same problem, 'diarrhoea', were noted on two separate occasions, without any intervention being suggested. A conception is understood as something the patient believes or thinks about his/her illness, treatment, medication or health status. The term 'conception' is not used in the documentation. Instead terms such as 'think', 'believe' and 'do not believe' are used to describe the patient's perspective. The patient referred to below was examined for liver problems and also had an undefined pain in one knee. The documentation states:

The patient is very concerned about her knee and believes that the pain is due to a leakage of bile. 'If the pain in my knee goes away, I will not require any pain relieving medication, and as a result my liver will get better'. (record 3)

The patient's conception 'that the pain is due to leakage of bile' is not commented on by the nurse. Interestingly, a quote is used to record the patient's words. However, the patient's argumentation is documented without further comment. Another example follows:

An attempt to do a lumbar puncture, the patient refuses to cooperate, he does not think that he is suffering from meningitis, he says that he has a urinary tract infection. (record 2)

These conceptions seem to be recorded because the nurses wish to highlight specific problems related to the patient's attitude. On two occasions, the conceptions are followed by interventions where the nurse administers medication. Intervention in the form of an 'explanation' was only provided on one occasion.

\section{Experiences of bodily changes}

There are seven notes about patients' comments on experiences of bodily changes (Table 2) from seven different patients. The notes concern patients' comments on the reaction of their body to the disease, medication or treatment. In the following extract, documented in the evening time, a patient was undergoing treatment for a serious form of liver disease, and a liver transplantation was under consideration.

The patient seems to be happy and content, is watching TV and has asked to be weighed, $56.3 \mathrm{~kg}$. The patient thinks that his legs are less swollen now. (record 4)

The nurse seems to relate the weight to the patient's comments on the decrease in swelling. The documentation does not reveal anything about the nurse's standpoint or the reason for the patient's request to be weighed. Another example:

The patient becomes frightened when she looks in the mirror and recognizes the Brillen Heamatoema. (record 11)

The experiences documented reveal something about the patient's interpretations of bodily changes. The notes were followed by interventions in terms of medication on two occasions, by 'moral support' on one occasion and by checking the pulse rate on yet another occasion.

\section{Areas identified indicating nurses' teaching interventions}

Five areas were identified in the documentation indicating teaching interventions:

- Information

- Motivating conversations

- Explanations

- Instructions

- Setting expectations

\section{Information}

Notes about nurses provision of information were identified on 15 occasions during the 206 days and concerned eight patients (Table 2). The term 'information' is used in all these notes. Information in the sense of conveying a message to the patient occurs on six occasions. These messages usually concern treatments, tests and planned examinations, e.g.:

The patient is informed about the need to collect urine over a 24-hour period (record 29)

The term 'information' is also used on two occasions as a reminder about something that the nurses might otherwise forget, e.g.: 
Inform the occupational therapist tomorrow. (record 4)

Yet another example is documentation on the distribution of written information in the form of leaflets. Comments on physicians' informative actions are noted on six occasions. One example is as follows:

Dr. NN had informed the patient about different alternative treatments. The patient was to think it over (treatment A or B) during the weekend. (record 6)

After the weekend the following was documented:

The patient has now returned. Everything went well at home. The patient is in favour of treatment $\mathrm{A}$, but has not yet reached a decision. The patient will discuss this matter with Dr. NN again. (record 6)

This note is the only one in the documented data that reveals an explicit intention to seek the patient's informed decision. Furthermore, there is one note about information to relatives. No documentation on nursing outcomes exists.

\section{Motivating conversations}

Motivating conversations concern treatments, medication and patients' experiences of bodily changes. A motivating conversation was identified on five occasions (see Table 2). Three of the five entries concern physicians holding motivating conversations with patients, e.g.:

Dr NN discusses a possible intrapleural catheter. The patient wants to think about it. (record 5)

The other notes consist of vaguely formulated phrases by which the nurse explains the reason behind the planned interventions or other aspects relevant to the patient.

I talked to the patient for a while. (record 4)

I sat down and talked to the patient. (record 21)

In one case, more definite terms are used:

The patient needs motivation and encouragement. (record 11)

The following was noted in another patient record under the heading of nursing status:

The patient asks for a sedative. She wants it because she considers that she has had a hard time since this morning and is irritated about things she does not want to discuss now. (record 4)

Under the heading of nursing intervention:

I tried to talk to the patient for a while. However, she was not satisfied with that and therefore she is given sedative medication. (record 4)
The documentation does not reveal what 'talk', means. Furthermore, the note 'she is not satisfied' implies that the patient said something, which is not included in the note. However, it shows that the nurse devoted time to the patient. In total, nursing outcome was noted on two occasions; one concerning the effects of medication and the other dealing with the effects of a conversation with the physician.

\section{Explanations}

Explanations occur on five occasions in the documentation and involve four patients (Table 2). The term 'explanation' is used in the notes to explain treatment and doses of medication. In the following example, a patient is angry because she has not been allowed to rinse her mouth with juice. The notation reads:

I tried to explain why I (the nurse) refused to give her (the patient) juice when she (the patient) is starving. (record 4)

Possible reasons for refusing the patient juice are not documented, and the patient's knowledge needs are not identified. Another example: under the heading of nursing status, the following was noted:

The patient is unable to sleep. He accepts that he cannot have more sleeping pills. The patient is afraid that lack of sleep may have serious consequences. (record 17)

Under the heading of nursing intervention:

I explain to the patient that a sleepless night is not harmful.

Under the heading of evaluation:

Is satisfied with this.

In this example, the explanation was documented as an intervention and evaluated. Nursing outcomes are noted in four of the five entries, e.g. 'the patient is still angry' and 'the patient fell asleep'.

\section{Instructions}

Instructions are related to the nurse's aim to help the patient to manage practical tasks. There are three notes in the documentation dealing with planned instructions concerning three patients (Table 2). Two examples follow:

Instructions about the Pariboy technique (breathing help). (record 25)

We will start training him to inject himself.(record 6)

The term 'instruction' is used once. The notes are documented under the heading of 'nursing status' but not followed by further comment under the heading of 'nursing interventions'. No nursing outcomes are noted. 


\section{Setting expectations}

There are three notations in the documentation concerning setting expectations in terms of exhortation or advice to a patient involving three patients (Table 2). One note concerns advice to 'drink more' while, in another, the patient is advised to stay in bed because of dizziness. The note below deals with a patient suffering from diarrhoea:

The patient did not receive any diet restrictions from the emergency ward. I advised the patient to fast, because eating immediately results in diarrhoea. (record 24)

It is worth noting that the note is documented under the heading of 'nursing status', thus not as an intervention. No nursing outcome is noted.

\section{The structure of the documentation}

Eight patient records contain no details of pedagogical relevance whatsoever. Although details of pedagogical relevance were identified in 27 of the 35 records, the analysis reveals that neither patients' need for knowledge nor teaching interventions were documented in a structured way. However, fragmented indications of patients' need for knowledge occur in the form of conceptions, questions and comments on their experiences of bodily changes. Terms and expressions indicating teaching interventions, such as information, motivating conversations, explanations, instructions and setting expectations, are recorded without being linked to a clear identification of patients' need for knowledge. Thus, according to the protocol developed by Ehnfors and Smedby (1993), in most cases the structure of the documentation corresponds to level one.

Another problem that was identified is the unclearness concerning the difference between 'nursing status' and 'nursing intervention'. Interventions of pedagogical relevance, for example 'explanations', were, in several cases, entered under nursing status.

No goals or nursing diagnosis of pedagogical relevance were found. Nursing outcomes were stated on some occasions but in relation to the evaluation of medical problems as opposed to a specific pedagogical problem. The most common second level keyword was 'psychosocial'. Thereafter, keywords with a physiological meaning were used, 'pain' being the most common. Keywords related to pedagogical activities such as 'cognition/development' (nursing status) and 'information/education' (nursing intervention) were not used in the nursing documentation.

\section{Discussion}

The results of this study show that the documentation of patient teaching in patient records is inadequate; terms and expressions are documented in a fragmented way and there is a lack of structure based on the nursing process. Patients' knowledge needs are implicitly stated in the conceptions, questions and experiences of bodily changes. The documented details appear to relate to informal teaching. Furthermore, teaching interventions are documented in an implicit way, such as information, motivating conversations, explanations, instructions and setting expectations. Only a few notations of pedagogical relevance were found in the records. Because of the lack of analysis of reasons and consequences in the notes, the documentation has a general character, with few terms and expressions of pedagogical importance. However, to be relevant, they must be related to a meaningful content and highlighted as a problem of pedagogical importance. As this is not the case here, both content and structure are obscure, and the documentation can, therefore, be deemed incomplete. Despite the fact that the documentation of nursing care in Sweden is a legal obligation (SFS 1985, SOSFS 1993) and that considerable research has been performed in the area (Ehnfors et al. 1991, Ehrenberg et al. 1996), this study reveals that the documentation of patient teaching is still poor.

The question can be asked whether there is a lack of adequate terms to describe patients' need for knowledge and nurses' teaching interventions. However, as described earlier, some established terms can be found in the VIPS model, such as keywords of pedagogical relevance (cognition/development and information/education) (Ehrenberg et al. 1996). Furthermore, there are several terms of pedagogical importance mentioned in North American Nursing Diagnosis Association (NANDA) International (2003). Thus, at least in theory, there are terms to guide the documentation process. The areas used as a guide (Friberg 2001, Gregor 2001) in this study have, to some extent, demonstrated how the content of teaching actions can be identified. However, terms used in everyday nursing conversations and the terms documented in patient records have more than one meaning and are context dependent (Ricoeur 1983). Consequently, the terms and expressions in the documentation are contextually bound, thus, the relevance of the interpretation must be judged by each individual concerned. The results from this study indicate that efforts must be made to elaborate on these terms to create clear documentation that indicates reflected recording of patient encounters. With reference to the keywords 'cognition/development' (nursing status) and 'information/education' (nursing interventions) in the VIPS model (Ehrenberg et al. 1996), the nurses must create an 
understanding about the terms and how to use them in relation to the patient's life-world.

Whether the terms and expressions employed in this study are valued by nurses as indications of patient teaching is also open to question. The documentation in patient records may not necessarily reflect what happened in the actual situation (Adamsen \& Tewes 2000). In the present study, the nurses used terms and expressions of pedagogical relevance but in a general way, indicating an unawareness of their relevance to patient teaching. For example, although the nurses documented patients' conceptions and questions, they did not seem to link them to expressions of a need for knowledge. The same phenomenon was observed in relation to teaching interventions. A term identified as an intervention, for example, 'explanation' is documented under the heading of 'nursing status'. The results also reveal that patients seek knowledge and that nurses use teaching strategies at night (see Table 2). Thus, there must be openness towards patients' knowledge needs both day and night. According to a study of nursing documentation (Karkkainen \& Eriksson 2003), the best documentation was found in the area of medical instructions and observations of vital functions. In a discourse analysis of nursing documentation, Heartfield (1996) argues that the patient is not viewed in an holistic way. Nursing documentation can be seen as a manifestation of and a part of the ritual of power relationships. According to the result of the present study, nurses document both their own observations and those of the patients concerning experiences of bodily changes with focus on the physical body. Barber-Parker (2002) stresses that nurses base their assessment on their assumption of the patient's learning needs rather than on the patient's actual learning needs. One reason for documenting patients' observations may be to give the patient a 'voice', which indicates a sense of responsibility for the patient's care. However, this is not synonymous with being open to the patient's need for knowledge. According to the results, patients' need for knowledge is not recognized in the same way as, for example, physical needs. As Maslow (1970) argues: 'To know and to understand' is a need among other needs. Neglecting a patient's need for knowledge, or interpreting it as something connected to a medical problem, reveals a reductionist stance. As Martin et al. (1999) state, it is difficult to evaluate the quality of the care provided if the documentation about the patient's health-care status is incomplete.

Nursing education must assume its responsibility for considering the patient in an holistic way, which also includes a learning aspect. Training to identify patients' need for knowledge and how to document it is crucial. Willingness to create pedagogical encounters, in which the patients' need for knowledge is fulfilled, must be encouraged. Casey (1995) recommends that more efforts should be devoted to the structure and design of patient records. Patients' needs, educational content and learner outcomes as well as nurses' lack of time are all aspects that should be taken into consideration. According to a recent study (Björvell et al. 2003), nurses regarded nursing documentation as beneficial to their daily work. The results of the present study reveal that it is necessary to include pedagogical aspects in the documentation. This highlights the importance of addressing not only formal and planned teaching but also informal and spontaneous teaching.

\section{Limitations of the study}

Only a limited number of records were analysed in this study, which implies that the results cannot be generalized in the traditional sense. Despite this limitation, the study has illuminated a field that has been poorly studied. An example of the contribution of this study is the development of a protocol for use in the analysis of the content of the documentation. Further research must be conducted to deepen the understanding of the documentation of pedagogical activities. The protocol described above must be further developed and evaluated in other contexts.

\section{Practical implications}

Both formal and informal patient teaching activities must be regarded as a regular part of everyday nursing practice. Patients' need for knowledge must be addressed in nursing assessments and everyday discussions about the patient's care. Discussions concerning teaching interventions must also form a part of the agenda. Pedagogical awareness must be created, which includes openness towards the patients' way of expressing learning needs and a willingness to use the documentation tools available. Therefore, nurses, health-care organizations and those responsible for nursing education must assume responsibility for documentation that is accurate and reflects nursing activities.

\section{Conclusion}

Terms and expressions indicating patients' knowledge needs and nurses' teaching interventions occur in the nursing records. However, because of the lack of structure based on the nursing process, the meaning of the content is obscure. Efforts must be directed towards elaborating on these terms as indications of patients' knowledge needs and nurses' teaching interventions. In addition, the pedagogical significance must be valued. Patients' need for knowledge is not 
valued in the same ways as other needs, such as physical needs. This can be interpreted as inability on the part of the nurse to identify patients' learning needs or to formulate nursing interventions in a reflected way. It can also be interpreted as uncertainty about how to use the documentation tools. It can be assumed that nurses and nursing students need education to develop their documentation skills, which in turn ensures high quality care.

\section{Contributions}

Study design: FF, A-LB, ML; data analysis: FF, A-LB and manuscript preparation: $\mathrm{FF}, \mathrm{A}-\mathrm{LB}, \mathrm{ML}$.

\section{References}

Adamsen L \& Tewes M (2000) Discrepancy between patients' perspectives, staff's documentation and reflections on basic nursing care. Scandinavian Journal of Caring Sciences 14, 120-129.

Bandura A (1977) Social Learning Theory. Prentice Hall, Englewood Cliffs, NJ.

Barber-Parker ED (2002) Integrating patient teaching into bedside patient care: a participant-observation study of hospital nurses. Patient Education and Counseling 48, 107-113.

Björvell C, Wredling R \& Thorell-Ekstrand I (2003) Prerequisites and consequences of nursing documentation in patient records as perceived by a group of registered nurses. Journal of Clinical Nursing 12, 206-214.

Cadd A, Keatinge D, Henssen M, O'Brien L, Parker D, Rohr Y, Schneider J \& Thompson J (2000) Assessment and documentation of bowel care management in palliative care: incorporating patient preferences into care regimen. Journal of Clinical Nursing 9, 228-235.

Casey FS (1995) Documenting patient education: a literature review. The Journal of Continuing Education in Nursing 26, 257-260.

Coates VE (1999) Education for Patients and Clients. Routledge, London and New York.

Coutts LC \& Hardy LK (1992) Teaching for Health. The Nurse as Health Educator. Churchill Livingstone, London.

Ehnfors M \& Smedby B (1993) Nursing care as documented in patient records. Scandinavian Journal of Caring Sciences 7, 209-220.

Ehnfors M, Thorell-Ekstrand I \& Ehrenberg A (1991) Towards basic nursing information in patient records. Vård $i$ Norden 21, 12-31.

Ehrenberg A, Ehnfors M \& Thorell-Ekstrand I (1996) Nursing documentation in patient records: experience of the use of the VIPS-model. Journal of Advanced Nursing 24, 853-867.

Ehrenberg A, Ehnfors M \& Smedby B (2001) Auditing nursing content in patient records. Scandinavian Journal of Caring Sciences 15, 133-141.

Ekman I \& Ehrenberg A (2002) Fatigued elderly patients with chronic heart failure: do patient reports and nurse recordings correspond? International Journal of Nursing Terminologies and Classifications 13, 127-136.

Falvo DR (1994) Effective Patient Education. A guide to Increased Compliance. Aspen Publishers, Gaithersburg Maryland.
Friberg F (2001) Pedagogiska möten mellan patienter och sjuksköterskor på en medicinsk vårdavdelning. Mot en vårddidaktik på livsvärldsgrund. Göteborgs universitet, institutionen för vårdpedagogik, Göteborg, Sweden (Pedagogical encounters between patients and nurses in a medical ward. Towards a life-world based didactics. Dissertation. Göteborg university, Department of health care pedagogics, Göteborg, Sweden).

Graneheim UH \& Lundman B (2004) Qualitative content analysis in nursing research: concepts, procedures and measures to achieve trustworthiness. Nurse Education Today 24, 105-112.

Gregor F (2001) Nurses' informal teaching practices: their nature and impact on the production of patient care. International Journal of Nursing Studies 38, 461-470.

Hale CA, Thomas LH, Bond S \& Todd C (1997) The nursing record as a research tool to identify nursing interventions. Journal of Clinical Nursing 6, 207-214.

Heartfield M (1996) Nursing documentation and nursing practice: a discourse analysis. Journal of Advanced Nursing 24, 98-103.

Idvall E \& Ehrenberg A (2002) Nursing documentation of postoperative pain management. Journal of Clinical Nursing 11, 734742.

Janz N \& Becker M (1984) Health belief model: a decade later. Health Education Quarterly 11, 1-47.

Karkkainen O \& Eriksson K (2003) Evaluation of patient records as part of developing a nursing care classification. Journal of Clinical Nursing 12, 198-205.

Marcum J, Ridenour M, Shaff G, Hammons M \& Taylor M (2002) A study of professional nurses' perceptions of patient education. The Journal of Continuing Education in Nursing 33, 112-118.

Martin A, Hinds C \& Felix M (1999) Documentation practices of nurses in long-term care. Journal of Clinical Nursing 8, 345-352.

Maslow AH (1970) Motivation and Personality, 2nd edn. Harper \& Row, New York.

Milazzo V (1980) A study of the difference in health knowledge gained through formal and informal teaching. Heart \& Lung 9, 1079-1082.

North American Nursing Diagnosis Association (NANDA) International (2003) Nanda Nursing Diagnoses: Definitions and Classifcation, 2003-2004. North American Nursing Diagnosis Association, Philadelphia.

Redman B (1993) The Process of Patient Education. Mosby Year Book, St Louis.

Ricoeur P (1983) Hermeneutics and the Human Sciences. Cambridge University Press, Cambridge.

SFS (1985) Patientjournallagen (The Patient Record Act) Svensk författningssamling. Liber, Allmänna Förlaget, Stockholm. p. 562 (in Swedish).

SOSFS (1993) Socialstyrelsens förskrifter. Socialstyrelsens allmänna råd. Omvårdnad inom hälso- och sjukvården. (General guidelines for nursing within the health and medical services). Socialstyrelsen Författningssamling, p. 17 (in Swedish).

Turner DS, Wellard S \& Bethune E (1999) Registered nurses' perceptions of teaching: constraints to the teaching moment. International Journal of Nursing Practice 5, 14-20.

Van Manen M (1991) The Tact of Teaching. The Meaning of Pedagogical Thoughtfulness. State University of New York Press, Albany.

Yura H \& Walsh MB (1988) The Nursing Process: Assessing, Planning, Implementing, Evaluating. Appleton \& Lange, New York. 